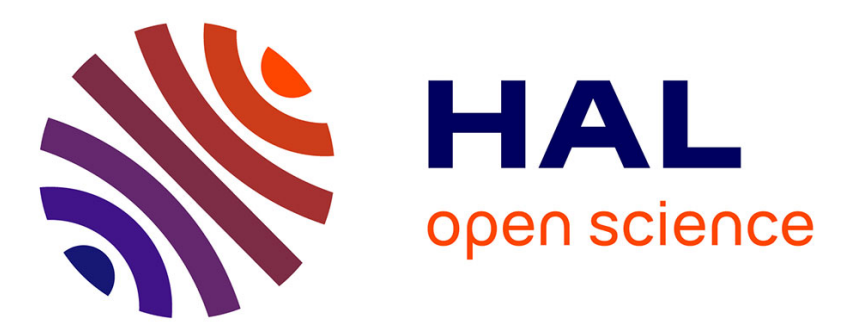

\title{
Injection and transport processes in organic light emitting diodes based on a silole derivative.
}

Nolwenn Huby, Guillaume Wantz, Lionel Hirsch, Laurence Vignau, A.S. Barriere, Laurent Aubouy, Philippe Gerbier

\section{- To cite this version:}

Nolwenn Huby, Guillaume Wantz, Lionel Hirsch, Laurence Vignau, A.S. Barriere, et al.. Injection and transport processes in organic light emitting diodes based on a silole derivative.. Journal of Applied Physics, 2006, 99, pp.084907. 10.1063/1.2190714 . hal-00186956

\section{HAL Id: hal-00186956 https://hal.science/hal-00186956}

Submitted on 13 Nov 2007

HAL is a multi-disciplinary open access archive for the deposit and dissemination of scientific research documents, whether they are published or not. The documents may come from teaching and research institutions in France or abroad, or from public or private research centers.
L'archive ouverte pluridisciplinaire HAL, est destinée au dépôt et à la diffusion de documents scientifiques de niveau recherche, publiés ou non, émanant des établissements d'enseignement et de recherche français ou étrangers, des laboratoires publics ou privés. 


\section{Injection and transport processes in organic light emitting diodes based on}

\section{a silole.}

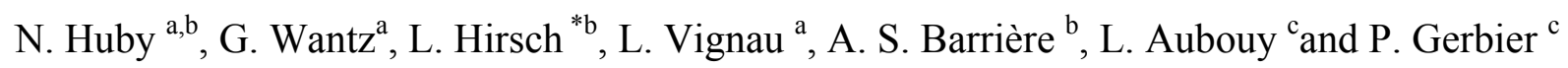

(a) Laboratoire de Physique des Interactions Ondes-Matière - PIOM (CNRS UMR 5501), ENSCPB, 16 Ave Pey Berland, 33607 Pessac Cedex, France

(b) Laboratoire d'Etude de l'Intégration des Composants et Systèmes Electroniques - IXL (CNRS UMR 5818), Université de Bordeaux 1, 351 cours de la Libération, 33405 Talence Cedex, France

(c) Laboratoire de Chimie Moléculaire et Organisation du Solide - CMOS (CNRS UMR 5637), Université de Montpellier II, CC 007, Place Eugène Bataillon, 34095 Montpellier Cedex, France

\section{Abstract}

This paper reports on the study of injection and transport of charge carriers in electroluminescent devices based on a novel highly emissive silole derivative 1,1-dimethyl2,5-bis(p-2,2'-dipyridylaminophenyl)-3,4-diphenylsilole. For that, current-voltage and luminance-voltage characteristics have been performed as a function of electron injection barrier height, and of temperature for ITO/PEDOT-PSS/silole/Au and ITO/PEDOTPSS/silole/Ca. The voltage dependence of current and luminance varies with the nature of the upper metal electrode. However, whatever the nature of the these, the hole contribution to the current reflects an easy injection at ITO/PEDOT-PSS/Silole interface. It is limited by the bulk of the emissive layer and corresponds to a hopping process. At high field a tunneling injection of electrons through a triangular barrier predominates for $\mathrm{Au}, \mathrm{Cu}$ and $\mathrm{Al}$ cathodes. In the case of a calcium cathode the observed currents are due to an easy injection of electrons fallowed

\footnotetext{
${ }^{*}$ Corresponding Author : hirsch@ixl.fr
} 
by a trapping process on coulombic centers and a field assisted thermal emission in the LUMO of the silole. These results prove the faculty of these molecules as both electron and hole transporting material. In order to fit the characteristics, we used classical theoretical models like Poole-Frenkel (P.F) and Fowler-Nordheim (F.N) theories. 


\section{Introduction}

Molecular compounds present a fascinating aspect since they have been used as organic semiconductors in light emitting diodes ${ }^{1}$. The different fields of research around the organic electronic allowed a better understanding of the phenomena inside the polymers or small molecules based electroluminescent devices. These improvements were possible thanks to the development of thin-film deposition technologies, but also thanks to the integration of the theoretical models for charges injection and transport through the organic layers. In this domain, the reported studies concern principally tris(8-hydroxyquinoline) and N,N'-diphenylN,N'-bis(3-methylphenyl)1-1'-biphenyl-4,4'-diamine (more fully known as Alq3 and TPD respectively) for the small molecules and poly(2-méthoxy,5-(2'-éthyl-héxoxy)-1,4-phénylène vinylène (MEHPPV) for the polymers ${ }^{2,3}$. Most of time, charge injection is believed to be a combination of tunnelling or thermionic injection, described by the Fowler-Nordheim (F-N) and Richardson-Schottky (R-S) equations respectively and space charge limited injection. Considering the F-N and R-S theories, different publications show that the models don't fit exactly the characteristics especially quantitatively, and propose new models ${ }^{4}$ or simulations ${ }^{5}$. We investigate and report the study of injection and transport in a novel and high efficient molecule named 1,1-dimethyl-2,5-bis(4-phenyl-di-2-pyridylamine)-3,4-diphenylsilole, (called silole in this paper). The silole-based compounds have occupied a growing position since Yamaguchi et al. ${ }^{6}$ firstly reported a general one-pot synthesis of 2,5-diaryl-3,4diphenylsiloles, and secondly, discovered their high efficiency in electroluminescence (EL) devices owing to their high electron transporting properties ${ }^{7}$. This property originates from a low-lying LUMO (Lowest Unoccupied Molecular Orbital), which is the consequence of an effective interaction between the $\sigma^{*}$-orbital of the silole silicon atom and the $\pi^{*}$-orbital of the butadiene fragment ${ }^{8}$. 
A novel silole derivative has been synthesized and characterized as emissive layer in electroluminescent device ${ }^{9}$. In order to understand the mechanisms associated with electrons and holes transport, we present in a first part the results of optical and electrochemical studies to describe the energetic schema of the structure, and then the current-voltage (I-V) and the luminance-voltage $(\mathrm{L}-\mathrm{V})$ characteristics at room temperature of different devices based on ITO (Indium Tin Oxide) /PEDOT-PSS (poly(3,4 ethylene dioxythiophene) doped with poly(styrene sulfonate))/ Silole/ Metal structure. The metals employed as cathode are calcium, aluminum, copper and gold. Moreover the temperature dependence of I-V and L-V were studied for devices with gold and calcium cathodes. Then we used theoretical models to describe I-V and L-V characteristics.

\section{Experimental}

Devices were fabricated on ITO-coated glass substrates (Merck, thickness $\approx 115 \mathrm{~nm}$, $\rho \approx 17 \Omega / \square$ ). Indeed, ITO is a commonly-used anode because it is transparent and has a high work function. A $50 \mathrm{~nm}$ layer of PEDOT-PSS was spun-coated at $5000 \mathrm{rpm}$ on top of ITO and annealed at $80{ }^{\circ} \mathrm{C}$ for about 1 hour. PEDOT-PSS is a conducting polymer acting, as a buffer, in reducing short circuit problems caused by the ITO deposition process. It weakly increases the work function of the anode and acts as a barrier to the oxygen and indium diffusion from $\mathrm{ITO}^{10}$. A $80 \mathrm{~nm}$-thick silole layer was then thermally evaporated under secondary vacuum $\left(p \approx 10^{-6}\right.$ mbar). Finally, a $90 \mathrm{~nm}$ of $\mathrm{Ca}(\mathrm{Al}, \mathrm{Cu}$ or $\mathrm{Au}$ ) layer was evaporated through a shadow mask on top of the silole. The devices have an active area of $10 \mathrm{~mm}^{2}$ and all steps of their preparation took place under controlled atmosphere. I-V and L-V were carried out in a liquid nitrogen cryostat under primary vacuum between $80 \mathrm{~K}$ and $300 \mathrm{~K}$. I-V characteristics were recorded using a Semiconductor Parameter Analyser Agilent 4156. Electroluminescent spectra were measuring within a CCD spectrometer (Ocean Optic PC 
2000). Absorption spectra were recorded on silole thin films deposited on glass substrates within a UV-visible spectrometer SAFAS Monaco 190DES. Finally, cyclic voltammetry was performed on a BAS 100 Electrochemical analyzer.

\section{Results and discussions}

\section{III.1. Energetic schema of the structure}

Preliminary studies were carried out in order to reach the energetic schema of the devices based on the silole derivative. First, cyclic voltammetry was performed on the silole in the liquid phase to estimate their highest occupied molecular orbital (HOMO) and lowest unoccupied molecular orbital (LUMO) energy levels. From the onset potentials of the oxidation and reduction processes, the HOMO and LUMO levels were calculated assuming the energy level corresponding to the electrochemical potential of the Calomel Electrode = $4.4 \mathrm{eV}^{11}$. They were then estimated to be $5.2 \mathrm{eV}$ and $2.8 \mathrm{eV}$ respectively. Consequently the energy difference, which corresponds to the bandgap $E_{g}$ of the silole, would be $2.4 \mathrm{eV}$.

In order to support this result, an optical absorption (OA) study has been performed. The OA spectrum is presented in figure 1B. In this case the estimated value of $E_{g}=2.5 \mathrm{eV}$ has been found, which weakly exceeds the previous one. At least, a normalized electroluminescent spectrum presented in figure $1 \mathrm{~A}$. shows that the emission peak is centered at $2.3 \mathrm{eV}$, which reveals the binding exciton energy in the silole. The chromatic coordinates of the electroluminescence emission, calculated on the CIE-1964 chromaticity diagram, are $\mathrm{x}=0.38$ and $\mathrm{y}=0.58$, and corresponds to a yellow-green color.

An energetic representation of the structure can then be achieved. It is represented in figure 2 .

The metals employed as cathode are calcium, aluminum, copper or gold ${ }^{12}$. The work function of the ITO/PEDOT-PSS is estimated at $5.1 \mathrm{eV}$ (est-ce que tu pourrais mettre une ref ?). Since the anode stays unchanged during all the following studies, the energetic barrier for holes 
injection is estimated at $\phi_{a}=\varphi_{\text {HOмо }}-\varphi_{\text {ITO/PEDOT-PSS }} \approx 0.1 \mathrm{eV}$. The data concerning the electrons injection barriers are reported in table 1.

\section{III.2. Current and luminance studies}

To evaluate the performance of the devices described above, I-V and L-V studies have been carried out under forward bias. In a first part, the electron injection through different barriers height is investigated. In a second part, the dependence in temperature for the two structures ITO/PEDOT-PSS/Silole/Au and ITO/PEDOT-PSS/Silole/Ca/Al is carried out.

\section{III.2.1. Influence of the nature of the cathode}

The I-V and L-V characteristics for the four investigated metals are represented in figure 3. They show clearly that the increase of the barrier height for the electron injection influences strongly the properties and the efficiency of the device. However, if $V_{a p}>V_{b i}$, seeing the $\phi_{a}$ value, which is the same for all the samples, the hole participation to the current must be unchanged whatever the nature of the cathode, for similar $\left(V_{a p}-V_{b i}\right)$ values.

In fact, for low $\left(V_{a p}-V_{b i}\right)$, the observed currents are quite similar. Consequently they must reflect hole transport exclusively. It is only from $V_{a p} \sim 5,12,14$ and $30 \mathrm{~V}$ for $\mathrm{Ca}, \mathrm{Al}, \mathrm{Cu}$ and Au cathodes respectively that the currents differ, indicating the participation of electrons. This is supported by the fact that it is also from these applied voltages, called turn-on voltages $\left(V_{t o}\right)$, that an electroluminescence becomes detectable. This proves that, electrons and holes necessarily cohabit in the silole layer, from these $V_{t o}$ values. In order to determine the electrical transport mechanisms, investigations as a function of temperature have been achieved and presented in the following paragraph. 


\section{III.2.2. Electrical transport mechanisms as a function of temperature}

We limited our extended study in the two extreme cases, i.e. with gold and calcium cathode. The cases of $\mathrm{Al}$ and $\mathrm{Cu}$ electrodes are the subject of a last remark.

\section{III.2.2.1. ITO/PEDOT-PSS/Silole/Au structure}

Devices with gold cathode offer interesting I-V and L-V characteristic networks represented in figure 4. I-V characteristics reveal two very distinct domains of voltage and temperature dependence. The first one (domain I) corresponds to $5 \mathrm{~V}<V_{a p}<25 \mathrm{~V}$. The second one (domain II) corresponds to $30 \mathrm{~V}<V_{a p}<40 \mathrm{~V}$.

\section{(i) Domain I}

Between $5 \mathrm{~V}$ and $25 \mathrm{~V}$ currents are weakly thermally activated. They increase by only one order of magnitude from $T=89 \mathrm{~K}$ to $293 \mathrm{~K}$ at a constant voltage. At low field and considering the energetic barrier for electrons $\phi_{c}$ close to $2.3 \mathrm{eV}$, an electron injection is excluded. By the way, no electroluminescence is observable. Then, we assume that only thermally activated hole injection can be considered. The activation energy has been calculated from Arrhenius plots. We found $W \approx 50 \mathrm{meV}$. This result is comparable with the potential barrier $\phi_{a}$ for holes at the anode/HOMO interface. For an interface ITO/silole it should be fairly $200 \mathrm{meV}$. With a PEDOT-PSS layer, it is reduced and should be smaller than $100 \mathrm{meV}$. In this case, we could admit that on the least resistance paths, the limitative phenomenon should be the hole injection. It is the reason why, as in a Schottky metal-semiconductor junction, the current should be given by ${ }^{13}$.

$$
J=A^{*} T^{2} \exp \left(-\frac{\phi_{a}}{k T}\right)
$$


With $A^{*}=\frac{4 \pi m_{h} q^{2} k^{2}}{h^{3}}$ the Richardson constant, $m_{h}$ the effective mass of the holes, $k$ the Boltzmann constant, $h$ the Planck constant, $q$ the elementary charge and $T$ the temperature. However, in this model, the current is independent on the applied voltage whereas it is obviously wrong in our case (see figure 4). Then the current limitation must be due to the bulk of the silole layer.

$\mathrm{I}-\mathrm{V}$ curves are plotted in linear scale in figure 5. It appears that for $V_{a p}<12 \mathrm{~V}$, the voltage dependence of the currents is quasi ohmic. Such a behavior must correspond to a hopping process (a thermally assisted tunnel transfer) between nearest neighboring localized states ${ }^{14}$. In this case, the current should be then given by:

$$
J=B F \exp \left(-\frac{<\Delta E>}{k T}+2 \alpha R\right)
$$

where $B$ is a constant, $F=\frac{V_{a p}-V_{b i}}{d}$ (d being the thickness of the silole layer), $\alpha$ expresses the spatial decrease of the wave-function of a hole near one site, $R$ is the distance between two neighboring sites and $\langle\Delta E>$ the average difference of the energy between the occupied site and the empty state. In this case, the experimental value of $\langle\Delta E\rangle \approx 50 \mathrm{meV}$ agrees with those found in the literature for this transport mechanism ${ }^{15-17}$.

\section{(ii) Domain II}

For an applied voltage greater than $30 \mathrm{~V}$ the currents are practically temperature independent. In this case, they cannot be described by a thermally activated mechanism. Moreover, this mechanism appears at very high electric field $\left(F>10^{6} \mathrm{~V} \cdot \mathrm{cm}^{-1}\right)$ and the voltage dependence is very strong compared to the first domain since the current density increases by three orders of magnitude on a weak voltage range. Finally, considering L-V curves, light emission starts around $V_{a p}=33 \mathrm{~V}$ whatever the temperature. It means that in this domain, electrons and holes coexist in the silole layer. All these remarks are consistent with a tunneling injection of 
electrons from the gold cathode to the silole through a triangular potential barrier. It is quite understood that the barrier height is very high at the gold/silole interface $(2.30 \mathrm{eV})$. However, for $V_{a p}>30 \mathrm{~V}$, the width of the barrier is reduced to $6 \mathrm{~nm}$ and is not longer than $4.5 \mathrm{~nm}$ at $V_{a p}$ $=40 \mathrm{~V}$. Moreover, in the case of a triangular potential barrier, the average of the barrier height $\phi_{c} / 2$, no longer than $1.15 \mathrm{eV}$, is taken into account. To verify these suppositions, we assume that the current-voltage characteristics follow the Fowler-Nordheim tunneling equation ${ }^{18}$

$$
J_{F N}=\frac{q^{3} F^{2}}{8 \pi h \phi_{0}} \exp \left(-\frac{4}{3}\left(\frac{2 m_{e}}{\hbar^{2}}\right)^{1 / 2} \frac{\phi_{0}^{3 / 2}}{q F}\right)
$$

In this expression $\phi_{c}$ is the barrier height for electrons at the interface $\mathrm{Au} /$ silole and $m_{e}$ the effective mass of the electrons. Consequently, we plot $\ln \left(\frac{J_{F N}}{F^{2}}\right)$ versus $F^{-1}$ in figure 6. At high field, i.e. for $F>4 \times 10^{6} \mathrm{~V} . \mathrm{cm}^{-1}$, which corresponds to an applied voltage $V_{a p}>32 \mathrm{~V}$, a linear relationship is obtained. From the slope of the straight line and with $m_{e}=9.109 \times 10^{-31} \mathrm{~kg}$, we extracted the barrier height for electrons at the injection. We found $\phi_{c}=2.5 \mathrm{eV}$. This value is weakly greater than that deduced from our previous electrochemical measurements. However, considering the measurement uncertainties, this theoretical model agrees with the experimental characteristics and properly describes the injection of electrons through the triangular barrier at the gold/silole interface.

\section{III.2.2.2. ITO/PEDOT-PSS/silole/Ca structure}

$\mathrm{I}-\mathrm{V}$ and L-V characteristics of ITO/PEDOT-PSS/silole/Ca are shown in figure 7 for different temperatures. For this kind of structure, these curves present three distinct domains:

a) For $V_{a p}<V_{b i}$ :

Only leakage currents can describe the weak observed current values. Indeed the natural electric field in the silole layer, due to the Fermi level alignment in the OLED structure, prevents the transport of electrons and holes in the LUMO and HOMO respectively. 
b) For $V_{b i}<V_{a p}<V_{t o}=7 \mathrm{~V}$ :

The current looks like the one observed in domain I for the structure with a gold cathode. Note that the difference of one order of magnitude is due to the device fabrication and to organic compounds (PEDOT-PSS and silole) fluctuations. Thus, in this domain, the currents should present the same interpretation as in the previous case, i.e. limitation occurs by a hole hopping mechanism in the bulk of the silole layer.

c) For $V_{a p}>V_{t o}$ :

In this domain, we note that the currents are four orders of magnitude higher than those observed with a gold cathode, for similar $\left(V_{a p}-V_{b i}\right)$ values. However, we have previously seen that the hole current contribution is the same whatever the metal cathode. So, in this domain, the current is mainly due to electron migration.

The potential barrier at the silole/Ca interface is almost ohmic (see table 1). Consequently we suppose that the electron transfer limitation is due to the bulk of the silole layer. Figure 8 clearly shows that currents, as well as luminance, are temperature dependent. Activation energies, $W$, are calculated from the slopes of Arrhenius plots. $W$ is found to be close to some hundreds $\mathrm{meV}$ and is a function of $V_{a p}$.

From these remarks, we suppose that the electron transfer limitation is due to a trapping of charge carriers in coulombic centers. The emission in the LUMO is then a function of both temperature and electric field $F=\frac{V_{a p}-V_{b i}}{d}$. The Poole-Frenkel model ${ }^{19}$ should agree with our experimental data. In this case, currents should be described by:

$$
J=q N_{i} \mu F \exp \left(-\frac{W}{k T}\right)
$$

Where $N_{i}$ is the density of coulombic traps in the silole layer, $\mu$ the mobility of the electrons in the LUMO and :

$$
W=E_{i}-\beta F^{1 / 2}
$$


Here $E_{i}$ is the energy of traps with regard to the lowest level of the LUMO, $\beta=\sqrt{\frac{q}{\pi \varepsilon_{0} \varepsilon_{r \infty}}}, \varepsilon_{0}$ is the vacuum dielectric constant, $\varepsilon_{r \infty}$ is the high frequency relative permittivity of the silole. The linear dependence of $\log \left(\frac{J}{V}\right)$ versus $\left(V_{a p}-V_{b i}\right)^{1 / 2}$ shown in figure 9 agrees with equations 4 and 5. Moreover the linear dependence of $W$ versus $\left(V_{a p}-V_{b i}\right)^{1 / 2}$, plotted in figure 10 , also confirms equation 5. From the extrapolation of $W$ at zero field i.e. $\left(V_{a p}-V_{b i}\right)=0$, we deduce the average depth of the traps: $E_{i} \approx 490 \mathrm{meV}$. This value is largely higher than the potential barrier $\phi_{c}$ at the interface $\mathrm{Ca} /$ silole, which confirms that the current limitation is not located at this interface.

In summary, for $F \leq 6 \times 10^{-5} \mathrm{~V} \cdot \mathrm{cm}^{-1}$, below the turn on voltage, the potential barrier height for an electron to be extracted from a coulombic center is still about $400 \mathrm{meV}$. For this reason, the current is mainly due to hole transport as in domain I for an ITO/PEDOT-PSS/Silole/Au structure. On the contrary, for $V>V_{t o}$, the internal electric field in the silole layer is higher than $10^{6} \mathrm{~V} \cdot \mathrm{cm}^{-1}$ and the barrier height is reduced up to $100 \mathrm{meV}$ (see figure 10). In this case, the current is mainly due to electron transfer in the silole layer. As a consequence, electrons and holes coexist in the organic layer and radiative recombinations are observed (see figure 8).

Finally, we can remark that for $\mathrm{Al}$ and $\mathrm{Cu}$ cathodes, a luminescence is also observed from $V_{a p}=12 \mathrm{~V}$ and $14 \mathrm{~V}$ respectively. This indicates the coexistence of electrons and holes in the silole layer. However in these cases, the barrier height at the interface cathode/silole $(1.3 \mathrm{eV}$ and $1.7 \mathrm{eV}$ for $\mathrm{Al}$ and $\mathrm{Cu}$ respectively) is highly greater than the depth of the coulombic centers. Consequently, in these cases, the limitation of the electron transfer must occur at the cathode level. 


\section{Conclusion}

Investigation of transport and injection in a novel and highly electroluminescent silole derivative are reported. ITO/PEDOT-PSS/Silole/cathode devices have been studied using I-V$\mathrm{T}$ and L-V-T techniques. It has been reported that hole transport is limited in the bulk of the silole layer. Hopping mechanism (thermally assisted tunnel transfer between localized states) agrees with experimental data. All the studied devices have the same anode, i.e. hole transport had the same origin in all samples. Electron transport neatly depends on the nature of the cathode metal:

$\checkmark$ For Au cathode, electron transfer limitation is located at the silole/cathode interface. Fowler-Nordheim mechanism is consistent with experimental data.

$\checkmark$ For Ca cathode, electron transfer is bulk limited. Poole-Frenkel mechanism agrees with experimental data. Coulombic traps as deep as $490 \mathrm{meV}$, with respect of the LUMO level, have been determined using this model. 


\section{References}

1

2

3 Chem. Eur. J. 6, 1683 (2000)

7 Silicon Compounds vol. 3, (Wiley, Chichester 2001), p. 641.

C. W. Tang and S.A. Van Slyke, Appl. Phys. Lett. 51, 913 (1987).

I. D. Parker, J. Appl. Phys. 75, 1656 (1994).

J.M. Lupton, I.D.W. Samuel, Appl. Phys. 32, 2973 (1999).

M. A. Baldo and S. R. Forrest, Phys. Rev. B 64, 1 (2001).

U. Wolf, V. I. Arkhipov and H. Bässler, Phys. Rev. B 59, 7507 (1999).

S. Yamaguchi, K. Tamao, J. Chem. Soc., 3693 Dalton Trans. (1998).

J. Chem 28, 1086 (2004). $6555(1983)$

I. H. Campbell and D. L. Smith, Appl. Phys. Lett. 74, 561 (1999).

S. M. Sze, Semiconductor devices, $2^{\text {nd }}$ edition, ( Wiley Interscience 1981)

A. Miller and E. Abraham, Phys. Rev. 120, 745 (1960).

A. K. Jonscher, Thin solid film 1, 213 (1967).

N. F. Mott, Phil. Mag. 19, 835 (1969).

R. M. Hill, Phil. Mag. 24, 1307 (1971).

J. G. Simmons, J. Appl. Phys 34, 1793 (1963).

S. Yamaguchi, T. Endo, M. Uchida, T. Izumizawa, K. Furukawa and K. Tamao,

S. Yamaguchi, K. Tamao, in Z. Rappoport, Y. Apeloig (Eds.): Chemistry of Organic

L. Aubouy, P. Gerbier, N. Huby, G. Wantz, L. Vignau, L. Hirsch and J. M. Janot, New

A.V. Dijken, A. Perro, E.A. Meulenkamp and K. Brunner, Org. Elec. 4, 131 (2003)

J. L. Brédas, R. Silbey, D.S. Boudreaux and R. R. Chance, J. Am. Chem. Soc 105,

J. R. Yeargan and H. L. Taylor, J. Appl. Phys. 12, 5600 (1968) 


\section{Figure captions}

Figure 1: A) Normalized electroluminescent spectrum of ITO/PEDOT-PSS/Silole/Ca structure B) Normalized absorption spectrum of the silole in the solid state.

Figure 2: Schematic representation of the energetic structure of the investigated devices.

Figure 3: Current density-voltage and luminance-voltage characteristics for devices based on ITO/PEDOT-PSS/Silole/Metal, with Metal= $\mathrm{Ca}, \mathrm{Al}, \mathrm{Cu}$ and $\mathrm{Au}$. Open and full symbols corresponds to current density and luminance curves respectively

Figure 4: Current density - voltage and luminance - voltage characteristics of ITO/PEDOT$\mathrm{PSS} / \mathrm{Silole} / \mathrm{Au}$ device at several temperatures from $89 \mathrm{~K}$ to $236 \mathrm{~K}$.

Figure 5: Current - Voltage dependence, in linear scale, of ITO/PEDOT-PSS/Silole/Au device at several temperatures from $89 \mathrm{~K}$ to $236 \mathrm{~K}$. This voltage region corresponds to domain I.

Figure 6: Electric field dependence of the current density between $25 \mathrm{~V}\left(3.2 \times 10^{-9} \mathrm{~m} . \mathrm{V}^{-1}\right)$ and $40 \mathrm{~V}\left(2.10^{-9} \mathrm{~m} \cdot \mathrm{V}^{-1}\right)$ for several temperatures.

Figure 7: Current-density-voltage and luminance-voltage characteristics of ITO/PEDOTPSS/Silole/Ca devices. Temperature exploration is included between $90 \mathrm{~K}$ and $266 \mathrm{~K}$.

Figure 8: Plot of $\log (J / V)$ versus $V^{1 / 2}$ for ITO/PEDOT-PSS/Silole/Ca and for several temperatures. The built in potential, $V_{b i}$, is taken into account. 
Figure 9: Plot of activation energy versus $\left(V_{a p}-V_{b i}\right)^{1 / 2}$. The values (full circles) are deduced from Arrhenius plots. The straight line represents a linear fit. 
Table 1: Summary of the data for the investigated structures. $\varphi_{m}$ represents the work function for the different metals, $V_{b i}=\mathrm{V}_{\mathrm{i}}(\mathrm{ITO} / \mathrm{PEDOT})-\mathrm{V}_{\mathrm{m}}$ (cathode) the built in potential, $\phi_{c}=\varphi_{m}$ $\varphi_{L U M O}$ the energetic barrier height for electrons injection.

$$
\varphi_{m}(\mathrm{eV}) \quad V_{b i}(\mathrm{~V}) \quad \phi_{c}(\mathrm{eV})
$$

$\begin{array}{llll}\text { Ca } & 2.9 & 2.2 & 0.01 \\ \text { Al } & 4.2 & 0.8 & 1.3 \\ \text { Cu } & 4.6 & 0.4 & 1.7 \\ \text { Au } & 5.2 & -0.2 & 2.3\end{array}$

TABLE 1 Huby et al. 


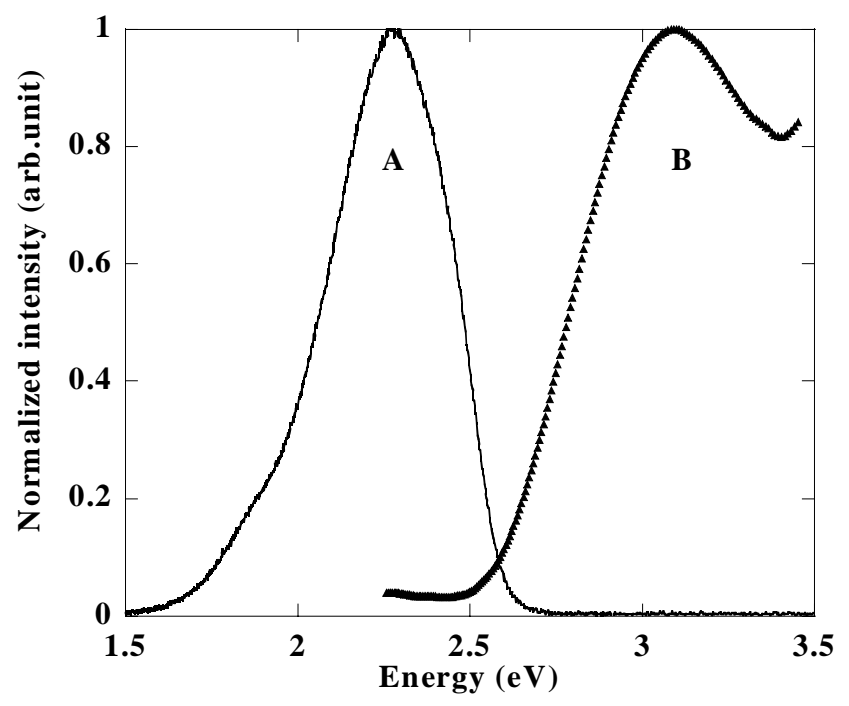

FIGURE 1 Huby et al. 


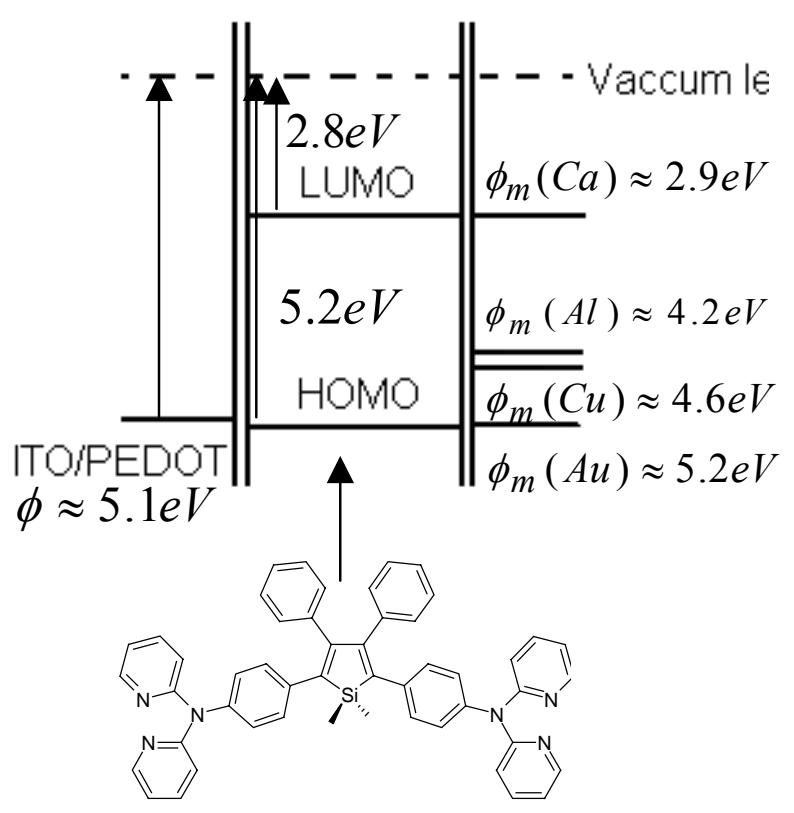

FIGURE 2 Huby et al. 


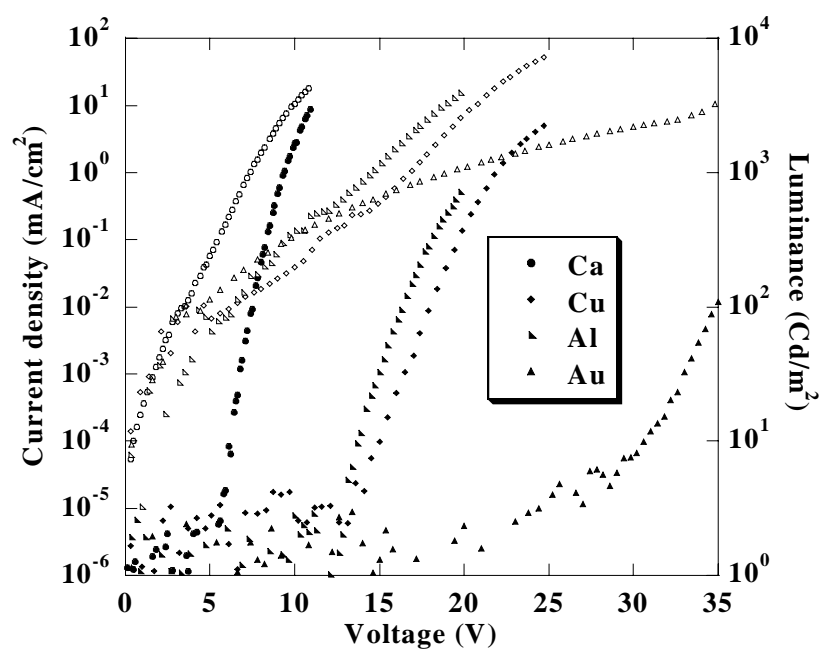

FIGURE 3 Huby et al. 


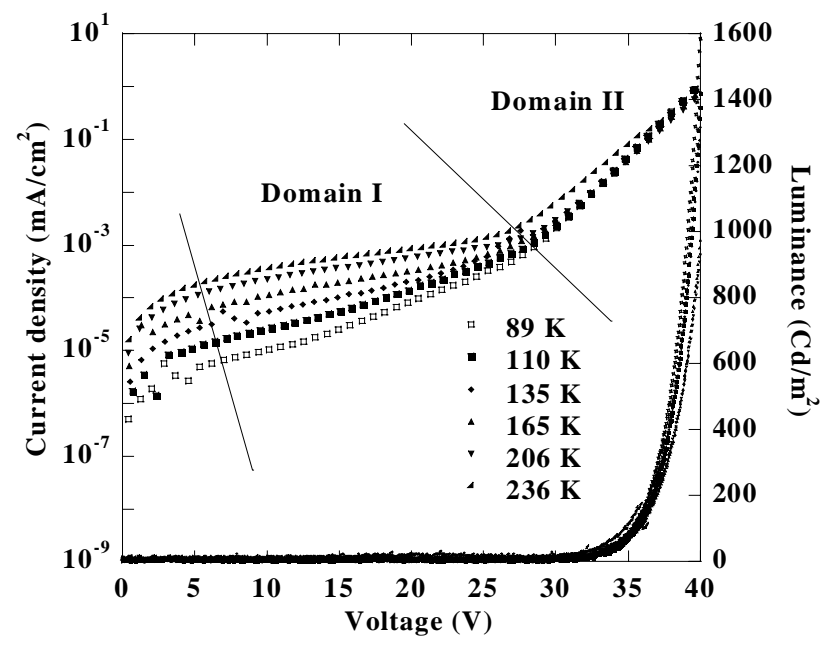

FIGURE 4 Huby et al. 


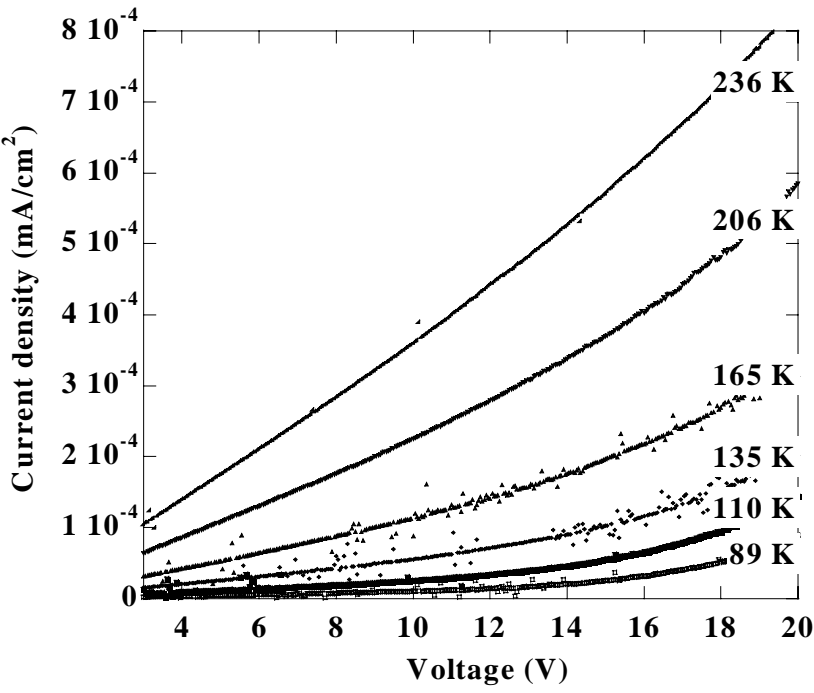

FIGURE 5 Huby et al. 


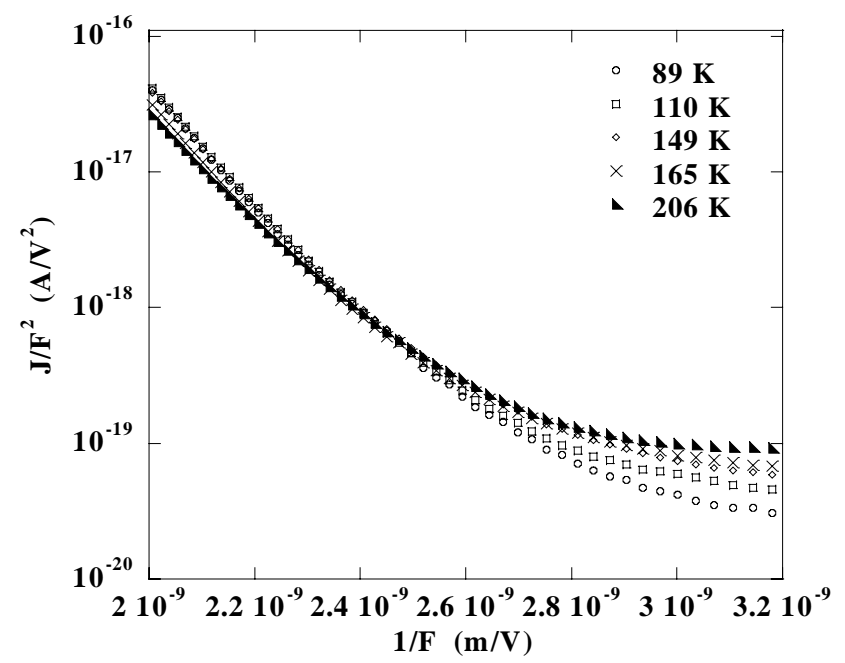

FIGURE 6 Huby et al. 


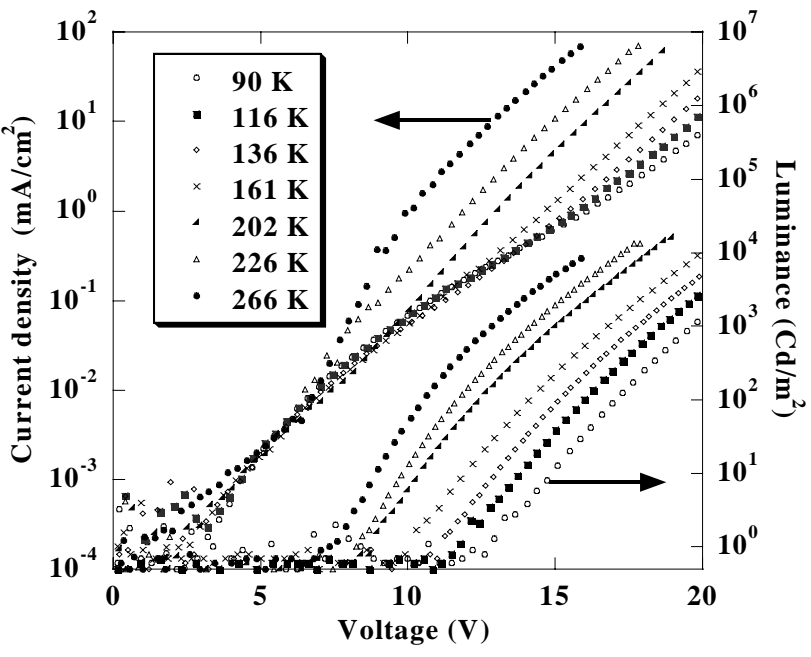

FIGURE 7 Huby et al. 


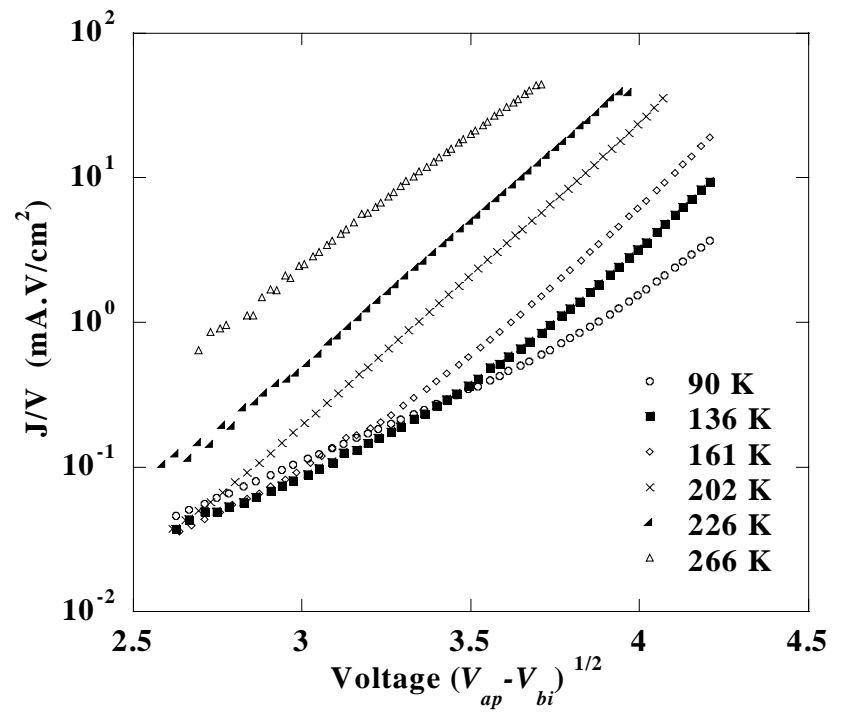

FIGURE 8 Huby et al. 


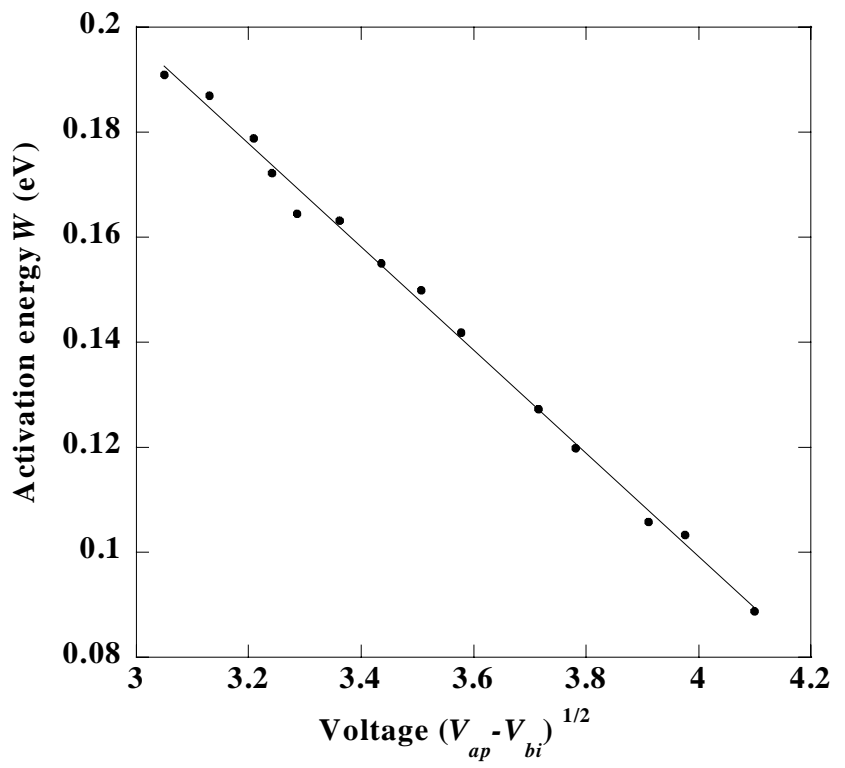

FIGURE 9 Huby et al. 